\title{
MAGNETIC FIELDS ASSOCIATED WITH SOLAR FLARES
}

\author{
EARLE B. MAYFIELD
}

San Fernando Observatory, The Aerospace Corporation, El Segundo, Calif., 90045, U.S.A.

\begin{abstract}
An investigation of strong magnetic fields associated with flares was made with the $61 \mathrm{~cm}$ vacuum telescope and spectroheliograph at the San Fernando Observatory. Magnetograms of the longitudinal component of the field were made daily during the period of September 25-29, 1969. Observations were also made during this same period of the enhanced radio emission at $3.3 \mathrm{~mm}$ wavelength. On September 27 an importance class 3 or 4 flare occurred in the region studied. Total magnetic flux was determined for September 25, 26, 28 and 29 for the region which included the flare. In an area of about 190 arc s by 250 arc s the flux values for these dates were respectively 2.4, 2.5, 2.2 and $2.8 \times 10^{22} \mathrm{Mx}$. Following the flare of September 27 the flux decreased significantly. Magnetic energy change in the region of the flare can be determined if an appropriate height is known. Following Howard and Severny (Astrophys. J. 137, 1242, 1963) a height of $10^{9} \mathrm{~cm}$ was used. This yields a value of $5 \times 10^{31} \mathrm{ergs}$ for the decrease of magnetic energy in the longitudinal component of the field.
\end{abstract}

\section{Introduction}

A number of recent theories of solar flares have proposed that magnetohydrodynamic processes, primarily in or near the region separating magnetic fields of opposite polarity, are responsible for flares. These include Severny (1958, 1961, 1963), Jaggi (1963), Petschek (1964), Syrovat-skii (1966), Sturrock and Coppi (1966), Alfvén and Carlqvist (1967) and Sturrock (1968). Although these theories differ in specific details of pre-flare field configuration, on-set and energy transfer, there is agreement that significant field changes should be observed in the region associated with a flare. Several models have been used to explain the observed plasma instability and the release of flare energy in times of $10^{2}$ to $10^{3} \mathrm{~s}$. Although these models postulate initial conditions in the fields prior to a flare, e.g. large magnetic gradient at the neutral line, complex structure with interpolated regions of opposite polarity, etc., and predict certain observable characteristics, the experimental verification of any theory is difficult or equivocal.

Previous investigations of magnetic fields associated with flares have measured magnetic flux changes during times of major flares. These measurements of fields have included both longitudinal and transverse components of the field. The results have shown significant flux reductions following major flares and have been interpreted in terms of energy loss by the fields to flare energy. Reductions of magnetic energy of order $10^{31}$ to $10^{32}$ ergs have been estimated.

This investigation of magnetic fields was directed to determining field configurations and flux changes associated with active regions which were expected to flare. Such a region studied was associated with McMath plage region 10333 near Mount Wilson sunspot 17503 which had a central meridian passage on September 27. 3, 1969. An importance class 3 or 4 flare occurred in this region at NO7, EO2 on September 27, 1969 beginning at about 0345 UT. High resolution magnetograms of the longitudinal component of the fields were obtained daily during the period of September 25 
through 29. These were made with the $61 \mathrm{~cm}$ vacuum telescope and spectroheliograph at the San Fernando Observatory and have a seeing limited resolution of about 1 arc s. Simultaneous radio maps at $3.3 \mathrm{~mm}$ wavelength with a resolution of about 2.8 arc min were made. Regions of millimeter emission which have been previously reported by Mayfield et al. (1970) have been observed to be associated with the neutral line of primarily bi-polar magnetic fields and to have enhancements prior to major flares. For the flare of September 27 an enhancement of more than $10 \%$ in the millimeter emission over an undisturbed region of the disk was observed about $36 \mathrm{hr}$ prior to the flare. This region was near the neutral line of the magnetic field associated with the flare.

\section{Experimental}

The instrument used for this investigation was the vacuum telescope and spectroheliograph of the Aerospace Corporation San Fernando Observatory. Since the instrument has not been described elsewhere, a brief description is given.

The solar telescope is a Coudé system consisting of a primary 24 in. $(61 \mathrm{~cm})$ diam and a secondary 12 in. $(30 \mathrm{~cm})$ diam cassegrain which feeds a gregorian mirror. This three mirror configuration produces both a flat field and a stigmatic image with two image sizes. The primary image diameter is $115 \mathrm{~mm}$ and the secondary is $50 \mathrm{~mm}$. All three of the surfaces of the telescope are aspheric. The cassegrain figures are ellipsoidal for the first surface and hyperboloidal for the second surface. The gregorian is an ellipsoid. All of the mirrors are made of Cer-Vit to reduce thermal problems and coated with silver to enhance the reflectivity. A dielectric coating is applied over the silver to reduce oxidation. Four plane mirrors are used to direct the beam through the axles to the spectroheliograph. The entrance window is a plane surface and the exit window is a field lens which images the entrance pupil of the telescope on the grating of the spectroheliograph.

The spectroheliograph uses a Littrow mounting with a three element lens and a $6 \times 10 \mathrm{in}$. $(15 \times 25 \mathrm{~cm})$ grating of 1200 grooves $/ \mathrm{mm}$. Dispersion with the grating used in second order is about $1 \mathrm{~mm} / \AA \AA$. Slit lengths are $57 \mathrm{~mm}$ for $70 \mathrm{~mm}$ film format and there are two exit slits to obtain both polarized spectroheliograms simultaneously for magnetic field data. A spectrum line servo eliminates thermal drifts or displacement of the spectrum during scanning. An automatic exposure control is incorporated in the scan drive. Scanning is accomplished by rotation of the spectroheliograph about an axis located in the base normal to the optic axis. To accommodate for rotation of the solar image and to permit scanning in any direction the system can be rotated around the optic axis. Both the telescope and spectroheliograph have a focal ratio of $f / 20$.

For this investigation, the CaI $6102.7 \AA$ line was used. This line was selected because it is well suited for moderate field strength up to about $1500 \mathrm{G}$. It was also the one used by Leighton (1959) in his development of the method for magnetic field measurements and subsequent studies (Simon and Leighton, 1964). The exit slit of the spectroheliograph was positioned in the blue wing, $0.07 \AA$ from the line center. The blue wing was selected to reduce the contrast in the spectroheliogram pairs. Both 
entrance and exit slits were set to $60 \mu \mathrm{m}$ which, with the grating used, gave a scan time of typically $140 \mathrm{~s}$.

The spectroheliograms were recorded on S0392 film and processed to an average density of about 1.0 and a gamma of 4.0. Careful process control was maintained to insure uniform development and contrast of the two original films. Photometric density scales were recorded on each film for subsequent calibration. The solar spectrum in the region of $\lambda 6102$ was also photographed on each film to be used for reduction of the magnetic field film. One of these films was then contact printed to produce a transparent positive print. This print was processed to the same density as the original and to a gamma of 1.0. To produce the magnetic field film, this positive reversal film is placed in registered contact with the other original negative and a photographic copy made of the two. This photographic subtraction of the positive and negative transparencies cancels any density variations between the two oppositely polarized spectroheliograms which are not caused by magnetic fields.

The reduction of the data to obtain field strength is based on the photometric calibration and the line profile obtained from the spectrum. The film is read with a computer controlled precision cathode ray tube densitometer which scans the film and records the density for a matrix of points separated by $100 \mu \mathrm{m}$ and computes the field strength. At present, uncertainty in the method yields an error of about $\pm 85 \mathrm{G}$ in the determination of field strengths.

\section{Results}

Magnetograms were obtained daily during the period of September 25/29, 1969. For the flare of September 27, magnetograms were obtained about $8 \mathrm{hr}$ prior to and about $16 \mathrm{hr}$ after onset. However, the data for September 27 were not suitable for reduction because of instrumental errors. Figure 1 is a magnetogram for September 25 taken at 2200 UT. Region 10333 is located near the center of the figure, north is at the top and west is on the right. Positive fields out of the surface are lighter and negative fields into the surface are darker than the average, zero fields. Typical field strengths range from a minimum of about $85 \mathrm{G}$ to a maximum of about $1450 \mathrm{G}$. Although much stronger fields exist in the sunspot umbras, these regions are very underexposed in the original spectroheliograms and hence do not yield magnetic field data. Magnetograms for September 26, 28, and 29 are shown in Figures 2 through 4. Region 10333 can be seen in each of these. Its structure is primarily bi-polar but both polarities have a spiral structure which changes with each day. This region can be readily identified, however, after 4 days. Maximum field strengths measured in this region were as high as about $1450 \mathrm{G}$ but typically a few hundred gauss.

Radio maps at $3.3 \mathrm{~mm}$ wavelength were also obtained during the same period. These were made with the $15 \mathrm{ft}(4.57 \mathrm{~m})$ diameter radio telescope at Aerospace in El Segundo. The telescope has a half-power beam width of 2.8 arc min and is computer controlled to map the Sun in a flexible manner. For the data used in this study, the antenna mapped a matrix of $21 \times 21$ points centered on the disk. The emission of 


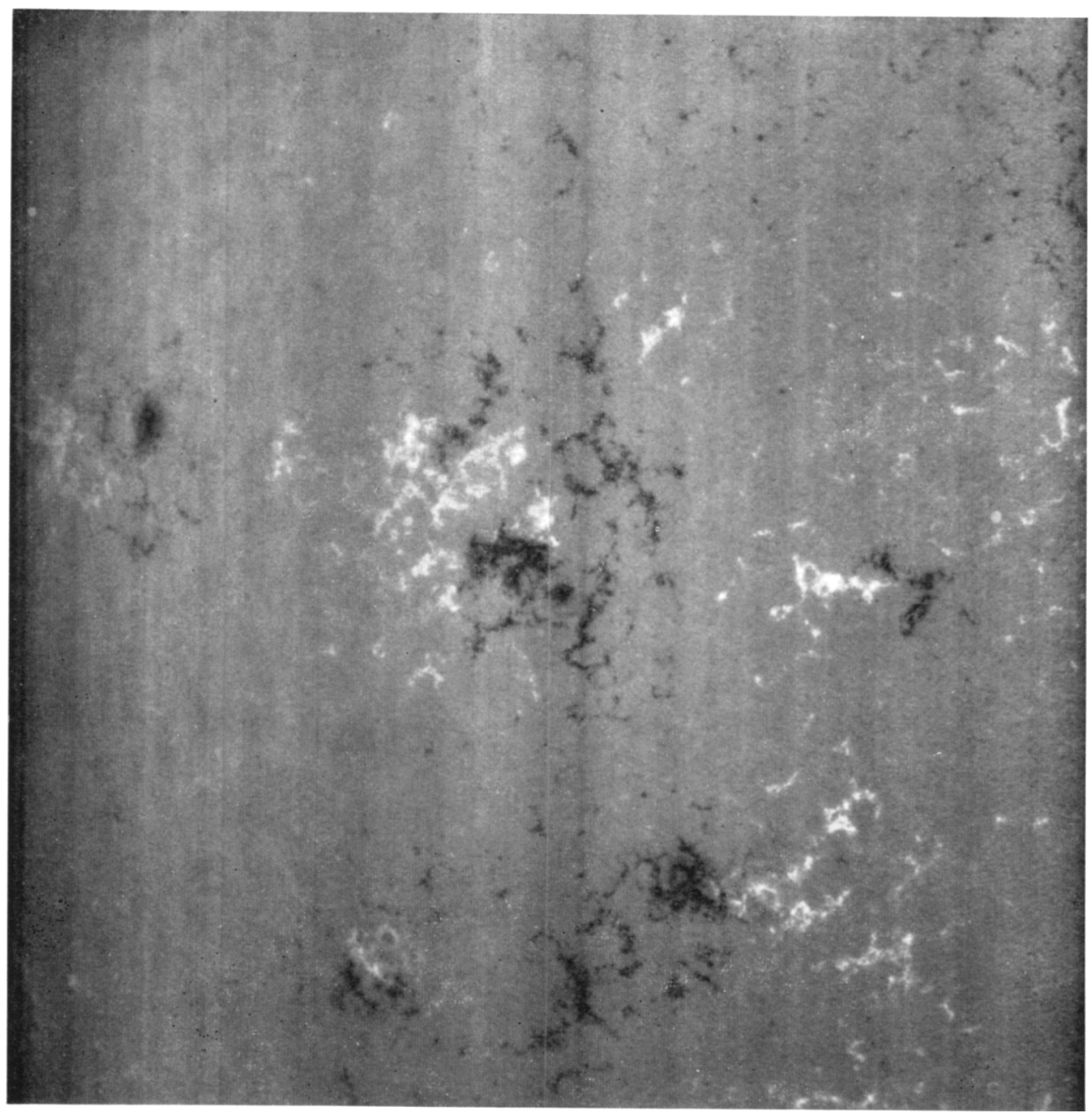

Fig. 1. Magnetogram of the longitudinal component of the fields for September 25, 1969. North is at the top, west is at the right.

each of these points was normalized to an undisturbed region near the center of the disk and the percent of enhancement of these points plotted. Isotherms were drawn on the map to identify enhanced regions of mm emission. Figures $5 \mathrm{a}$ and $5 \mathrm{~b}$ show $\mathrm{mm}$ maps taken on September 25 and 26 prior to the flare.

\section{Data Analysis}

The magnetograms were reduced with the cathode ray tube densitometer. For the pictures of September 25/29, an area of about 190 arc s $\times 250$ arc s was used. A total of $1.56 \times 10^{5}$ density values were read. To increase the signal to noise and to reduce the number of values for computation, each four adjacent points were averaged. 


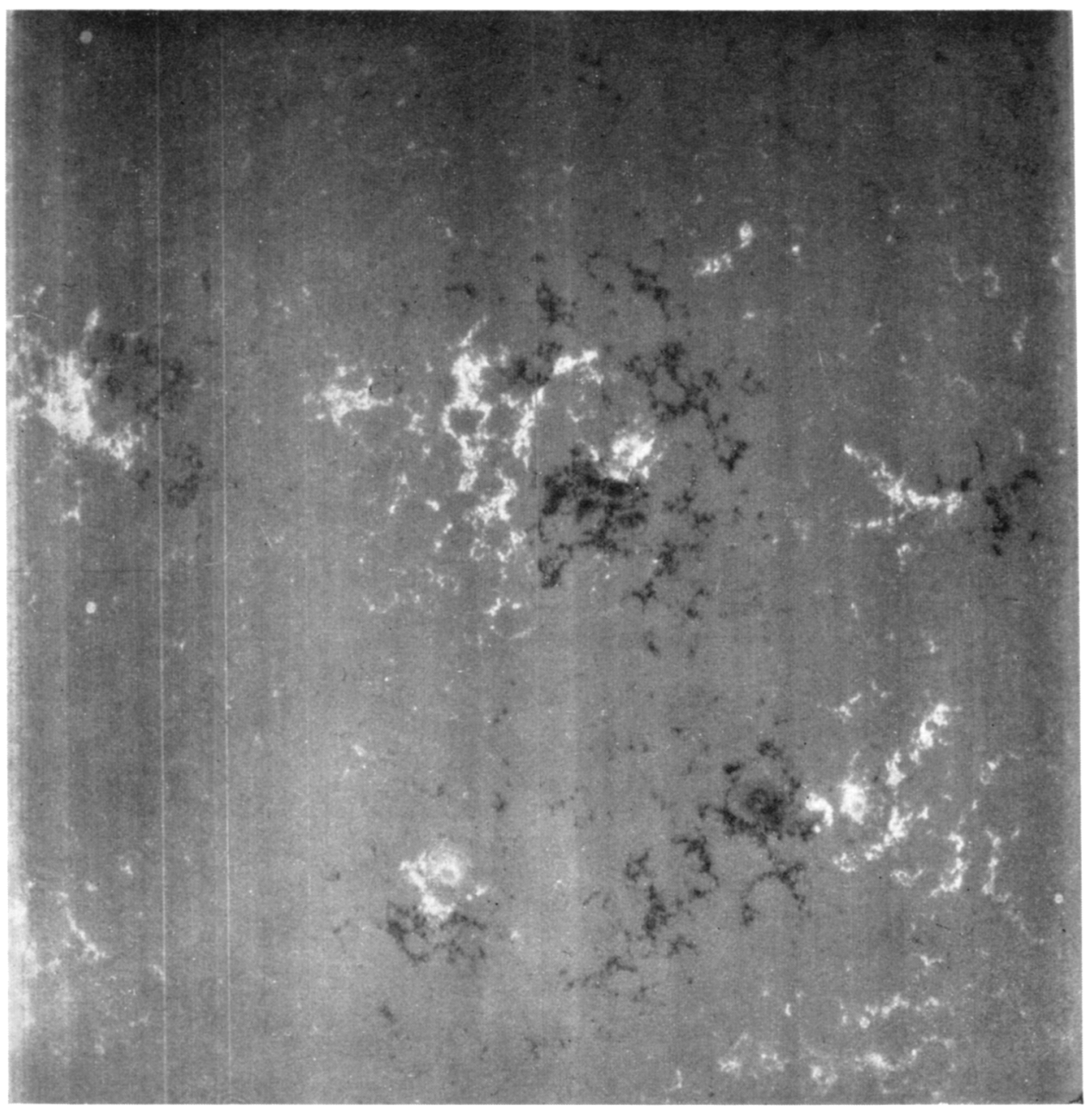

Fig. 2. Magnetogram of the longitudinal component of the fields for September 26, 1969.

This gave 40000 final values. The mean of these was determined and used as the average density corresponding to zero magnetic field.

Plots of the density data were made in frequency distribution histograms. Since the distribution is skew because of saturation in the densitometer only the low density half was plotted. This is shown in Figures 6a, b, c, and for d September 25, 26, 28 and 29. These show the frequency of occurrence of magnetogram density from the mean, zero field, to the minimum density corresponding to the maximum fields measured. It is apparent that significant changes in the distribution histograms occurred associated with the flare of September 27. The marked increase in frequency at the origin indicates a reduction in stronger fields. These distributions also show an increase in occurrence for densities associated with strong fields. This is caused by saturation of the line and 


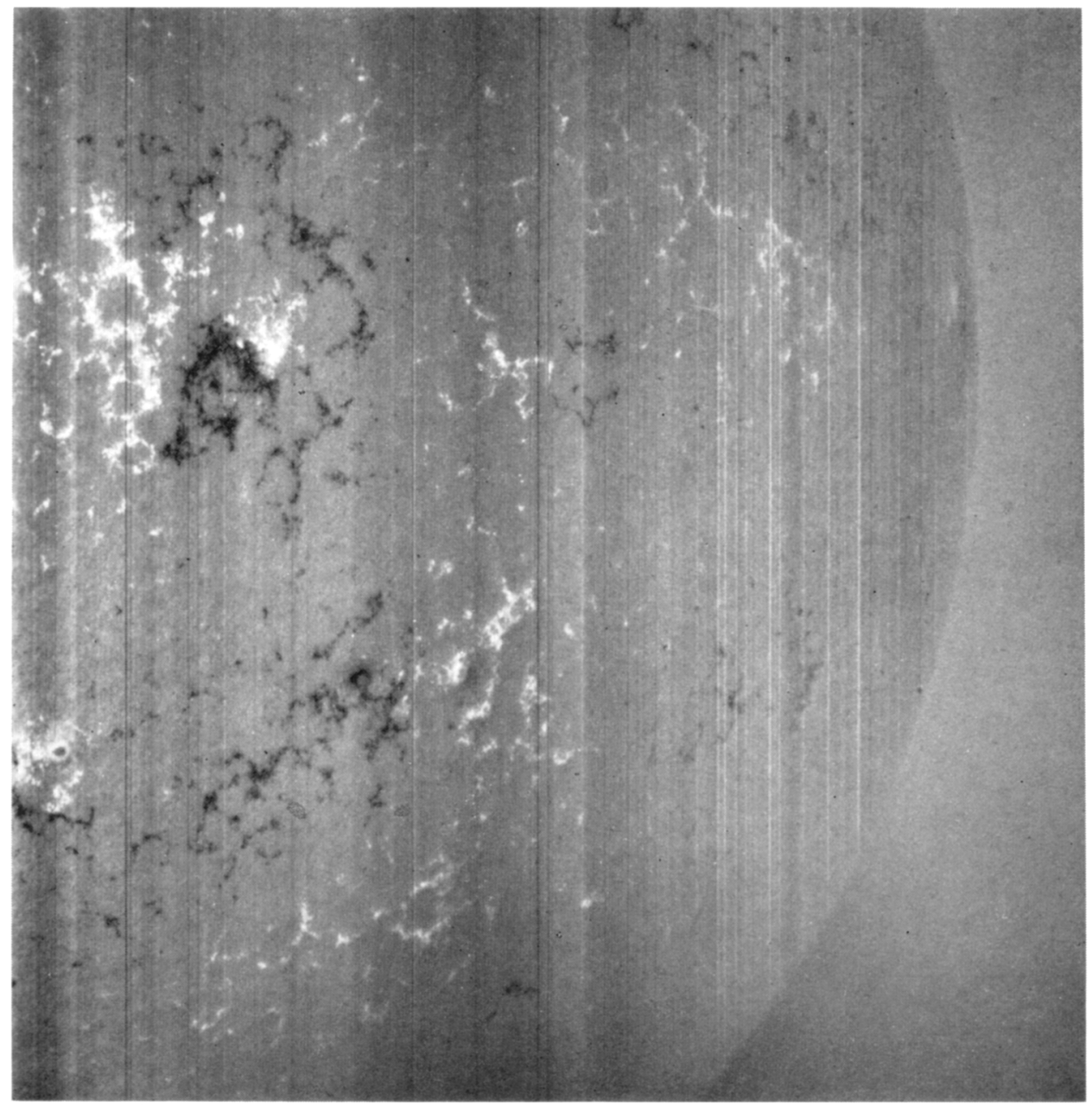

Fig. 3. Magnetogram of the longitudinal component of the fields for September 28, 1969.

by drifting of the line position and setting at the exit slit of the spectroheliograph. The calibration spectrum used to obtain $\mathrm{dD} / \mathrm{d} \lambda$ for field strength determination shows that fields of $\pm 1450 \mathrm{G}$ could be measured without saturation. This agrees well with Leighton's (1959) estimate of $\pm 1500 \mathrm{G}$.

Total magnetic flux was computed from the histograms and the area data determined from the known size of the densitometer spot on the original film. The flux for September 25, 26, 28 and 29 are shown in Table I.

These values show a decrease on September 28 following the flare of September 27. The general increase in magnetic flux of this region during the period was in agreement with other data which show that the region was growing and increasing in complexity. 


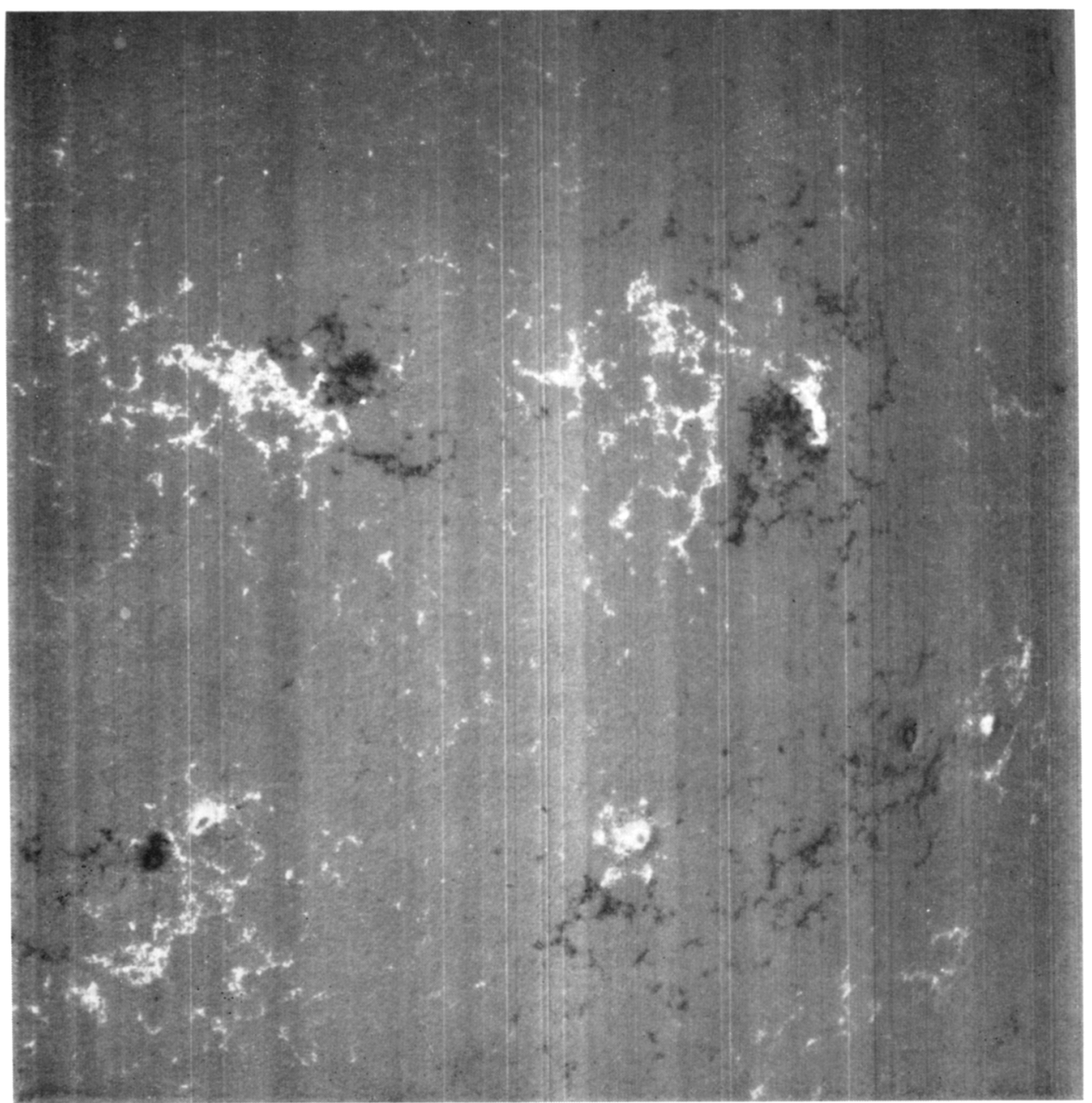

Fig. 4. Magnetogram of the longitudinal component of the fields for September 29, 1969.

\section{TABLE I}

Magnetic flux observed in an area of $190 \times 250$ arc s for September 25/29, 1969

\begin{tabular}{ll}
\hline Date & Magnetic flu \\
$9 / 25$ & $2.4 \times 10^{22}$ \\
$9 / 26$ & $2.5 \times 10^{22}$ \\
$9 / 28$ & $2.2 \times 10^{22}$ \\
$9 / 29$ & $2.8 \times 10^{22}$
\end{tabular}




\section{Discussion}

The flare of September 27 was a major event of geophysical significance. The ESSA Solar Geophysical Data (1970) gave an importance classification number of $3 \mathrm{~N}$ or 4B. It produced $X$-rays in the $0-20 \AA$ interval and a large increase in charged particles

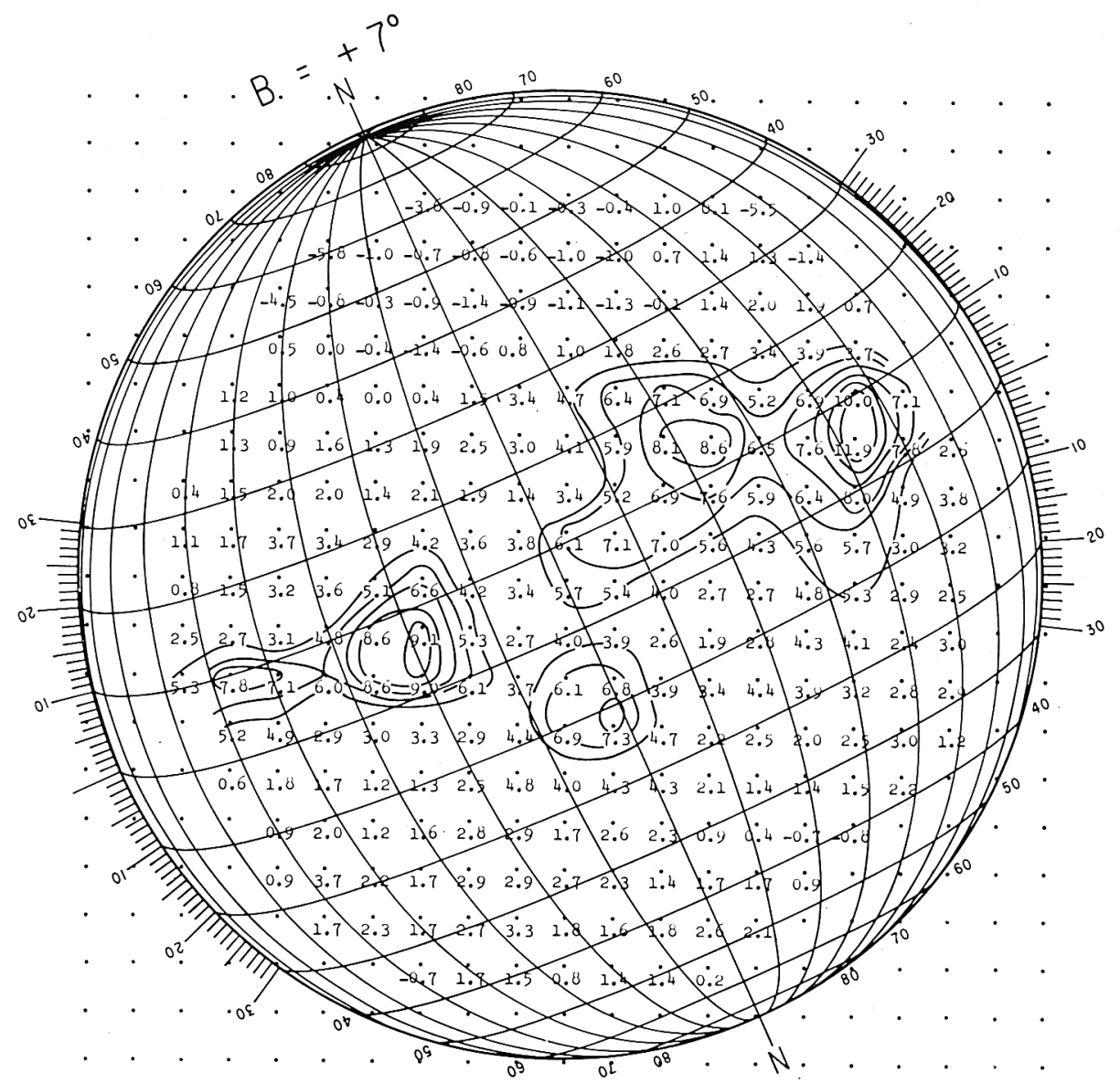

Fig. 5a. Map of $3.3 \mathrm{~mm}$ emission for September 25, 1969. Numbers are percent of enhancement over an undisturbed region, isotherms are for enhancements of $5 \%$ and greater for $1 \%$ increments.

for $E>10 \mathrm{MeV}$ at satellite altitudes. It also caused a large geomagnetic storm on September 29, 30 although the effects of the flare of September 25 may have contributed. The peak radio flux was reported as 2000 flux units $\left(1\right.$ unit $=10^{-22} \mathrm{w} / \mathrm{m}^{2} \mathrm{~Hz}$ ) and types II and IV bursts were recorded in $\mathrm{m}$ and $\mathrm{dm}$ wavelengths followed by a noise storm in meter wavelengths. 
The decrease in magnetic flux from September 26-28 appears to be associated with the flare on the 27th. Previous determinations of magnetic energy changes associated with large flares particularly those with charged particle emission have been reported. Evans (1959) observed magnetic field changes during an importance $1+$ flare on April 30 , 1959. Assuming a height of $5 \times 10^{8} \mathrm{~cm}$, an energy change of $4 \times 10^{31}$ ergs was

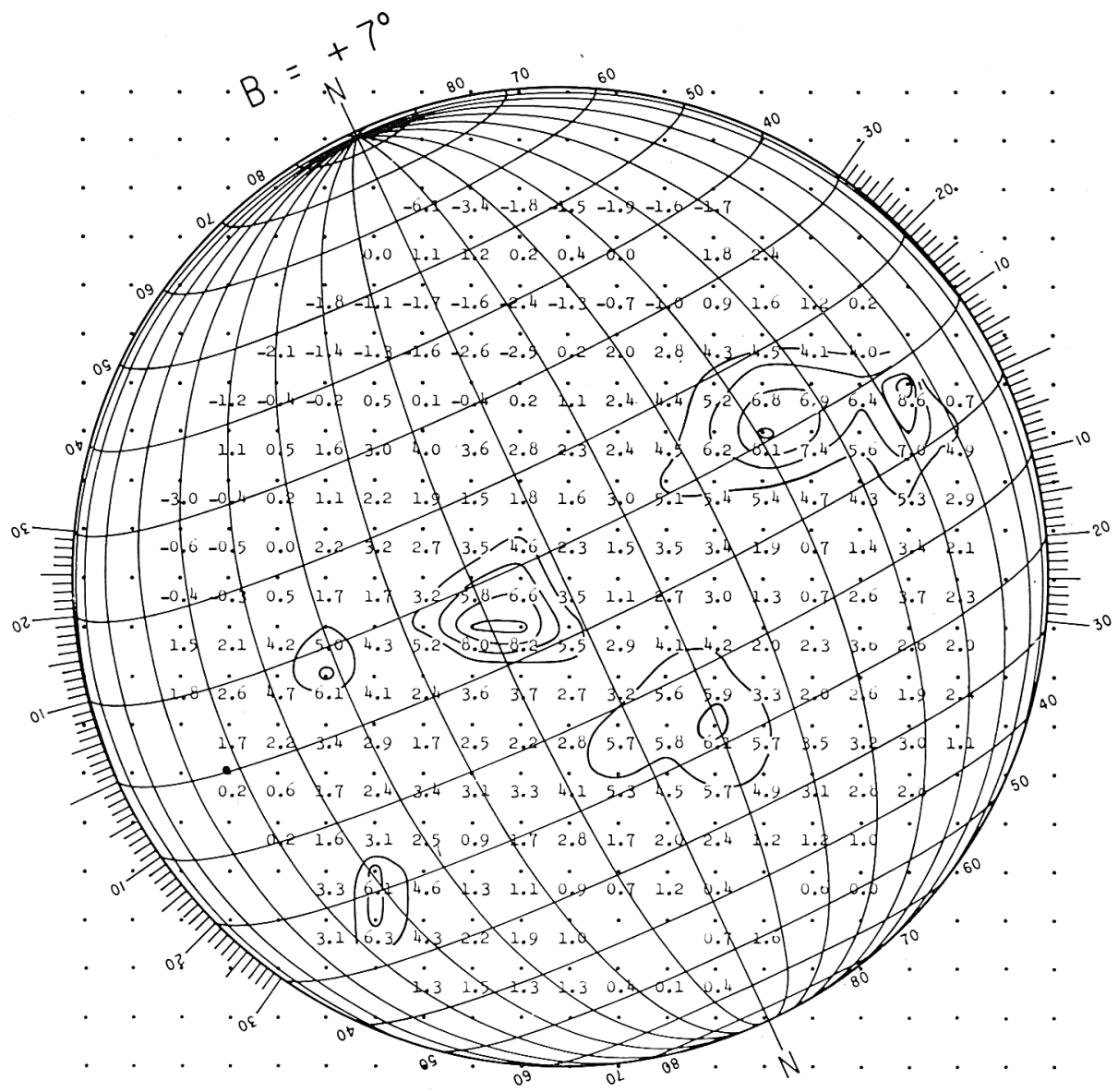

Fig. 5b. Map of $3.3 \mathrm{~mm}$ emission for September 26, 1969. Numbers are percent of enhancement over an undisturbed region, isotherms are for enhancements of $5 \%$ and greater for $1 \%$ increments.

determined. Gopasyak (1961) obtained a value of $10^{32}$ ergs for several importance class $2+$ or $3+$ flares. Howard and Severny (1963) have measured an energy of $4 \times 10^{32}$ ergs for the flare of July 16,1959 . This flare produced geophysical effects and charged particle radiation. Malville and Tanberg-Hanssen (1969) have speculated that energy of approximately $10^{31}$ ergs could have been dissipated by the magnetic 
field for the flare of May 21, 1967. Severny (1969) has measured energy changes in magnetic fields of about $5 \times 10^{32}$ ergs for the flare of July 7, 1966. These experimentally measured values of flare energy were obtained from observations of the longitudinal component of the magnetic field.

Determination of energy in the magnetic field requires an assumption of the effective height of the field. Following Howard and Severny (1963) a value of $10^{9} \mathrm{~cm}$ was used. This yields $5 \times 10^{31}$ ergs for the change in energy in the longitudinal component of the magnetic field for the flare of September 27. This change which occurred in

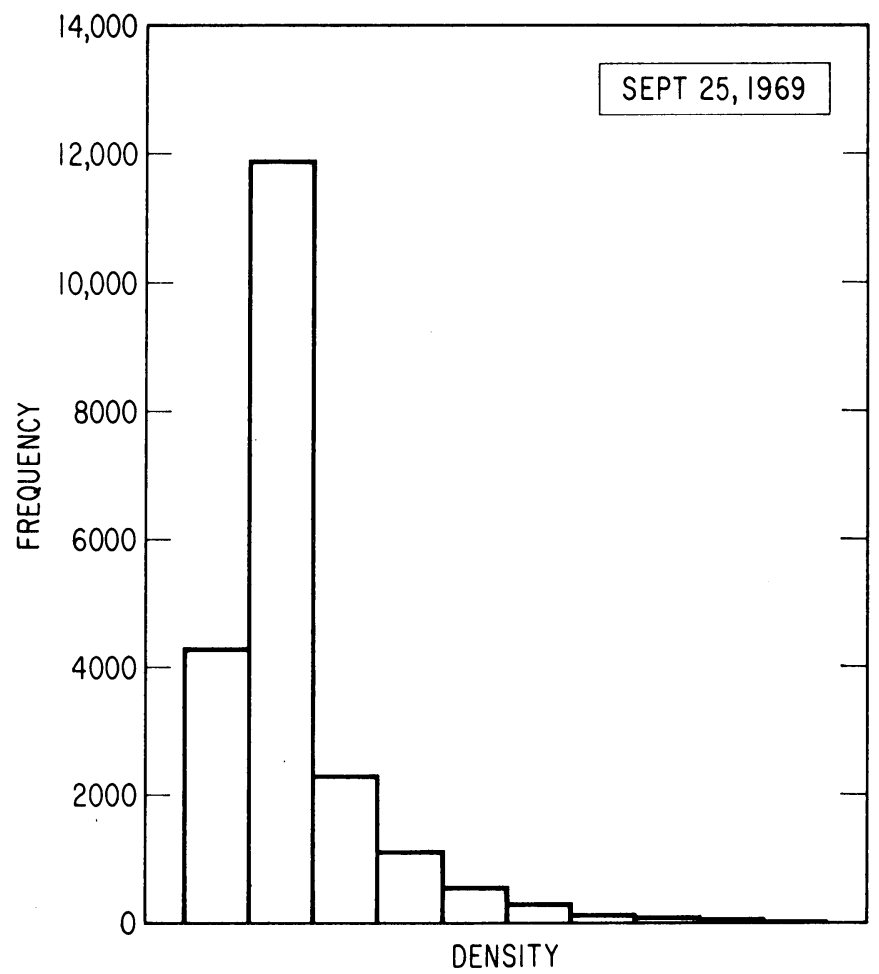

Fig. 6a. Frequency distribution histogram of film density for the positive field component of the magnetograms for September 25, 1969.

about $48 \mathrm{hr}$ was based on data taken $8 \mathrm{hr}$ before and $40 \mathrm{hr}$ after the flare. In the day preceding the flare the magnetic energy increased by about $10^{32}$ ergs. This is similar to the observations of Severny (1969) who noted that the energy typically increased before a flare. During the second day after the flare an increase in field energy was observed.

Estimates of energy change based on measurements of the longitudinal component of photospheric magnetic fields are subject to considerable uncertainty. Chromospheric currents significantly alter the assumed scalar potential as Mogilevsky and 
Shelting (1966) have shown. Solar rotation also leads to errors both in foreshortening of the area and change in the longitudinal field strength with aspect. Corrections can be made for foreshortening. However, corrections for field changes with aspect are questionable. The flare of September 27 occurred near central meridian and the magnetic field observations were made within $25^{\circ}$ of the center of the disk. This significantly reduced the problem of correcting for observations made near the limb.

As has been previously reported by Mayfield et al. (1970) there is enhanced radio emission at millimeter wavelengths about one day prior to importance class 2 or 3 ,

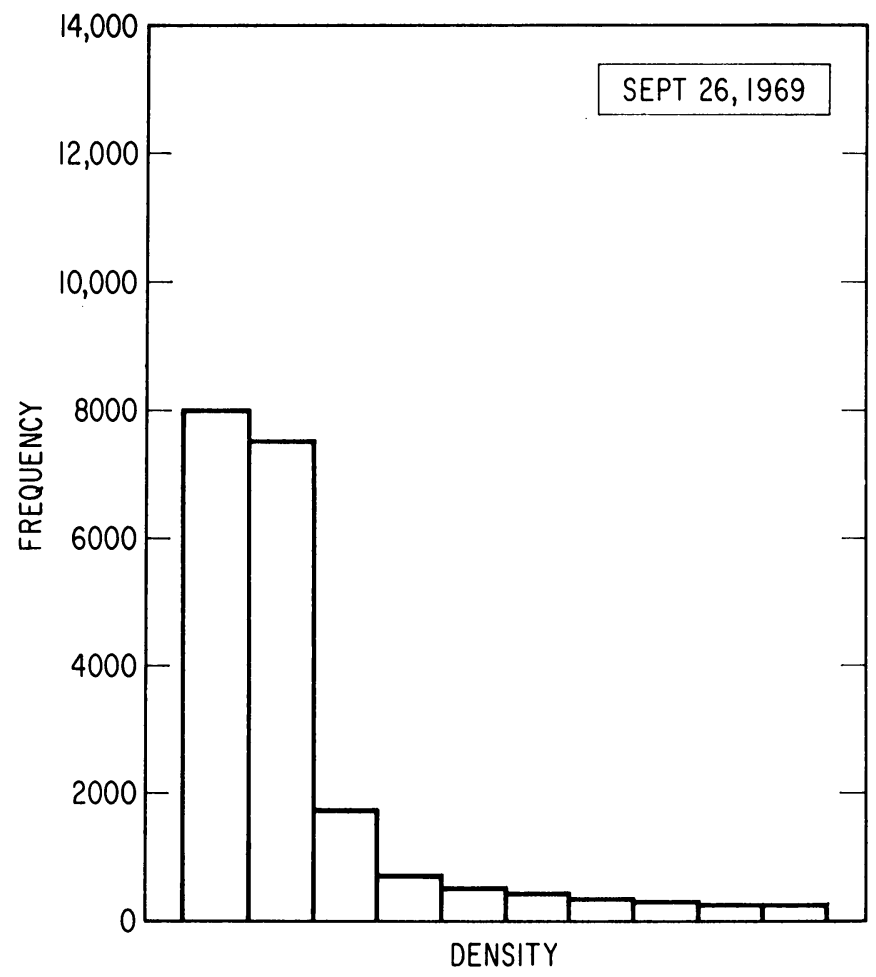

Fig. 6b. Frequency distribution histogram of film density for the positive field component of the magnetogram for September 26, 1969.

flares. This enhancement occurs near the neutral line of primarily bipolar magnetic fields and is generally confined to small regions of less than 3 arc min in extent. Typically the enhancement is greater than $8 \%$ and the temperature gradient is greater than $0.5 \% / \mathrm{deg}$. prior to these large flares. For the days of September 25 and 26 the enhancements were about $10 \%$ and the gradients greater than $0.5 \% / \mathrm{deg}$. The maximum coincided with the central region of the spiral field where the two polarities are contiguous. For September 26 the $8 \%$ isotherm conforms closely with the neutral line both in length and direction. However, lack of antenna resolution prevents identifying the shape with certainty. 
These observations of magnetic energy changes associated with a large flare accompanied by charged particle radiation are consistent with other measurements and with theoretical predictions. The occurrence of enhanced millimeter radio emission consistently observed to precede large flares is not explicitly predicted. Although the models proposed by Severny and by Jaggi can explain this observed heating at the neutral line, their analyses were made to explain instabilities associated with the flash phase of the flare which yield time constants of $10^{2}$ to $10^{3} \mathrm{sec}$. However, Furth et al. (1961) have considered instabilities in sheet pinches in laboratory plasmas and

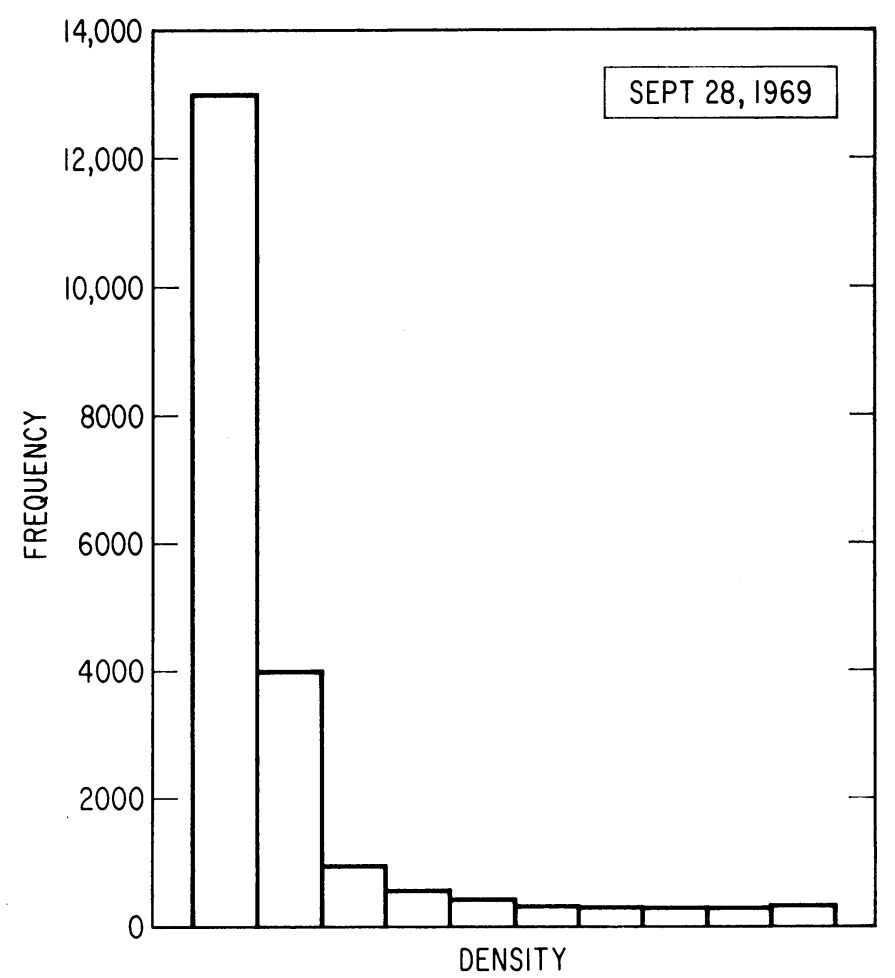

Fig. 6c. Frequency distribution histogram of film density for the positive field component of the magnetogram for September 28, 1969.

have shown the existence of several modes with characteristic time constants for local and non-local instabilities. The results of this investigation and previous results by Mayfield et al. (1970) suggest that similar processes may occur in flares.

\section{Acknowledgements}

The author thanks Dr F. I. Shimabukuro who kindly provided the $3.3 \mathrm{~mm}$ radio observations and Mr Dale Vrabec and Mr Neal Baker who collaborated in the 


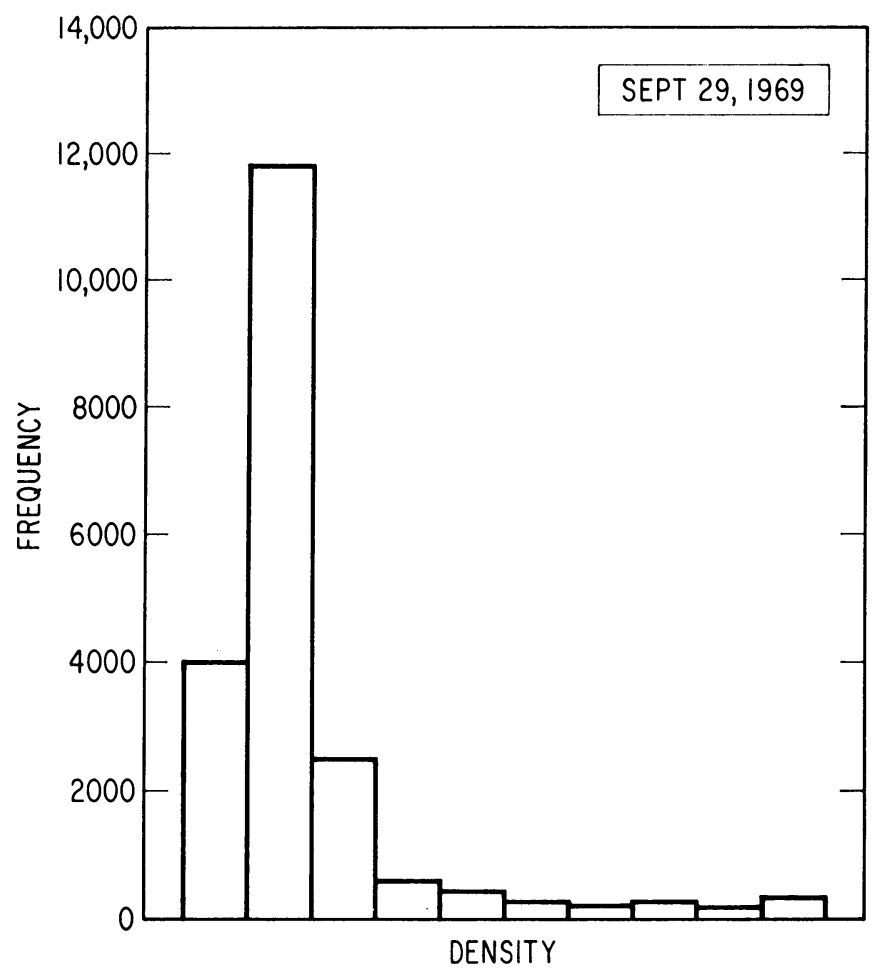

Fig. 6d. Frequency distribution histogram of film density for the positive field component of the magnetogram for September 29, 1969.

magnetic field measurements. Mr Kenneth Reed assisted with the densitometer reduction and numerical computations.

\section{References}

Alfvén, H. and Carlqvist, P.: 1967, Solar Phys. 1, 220.

ESSA Solar Geophysical Data: 1969, ESSA Research Laboratories, U.S. Dept. of Commerce, No. 307, Part II.

Evans, J. W.: 1959, Astron. J. 64, 330.

Furth, H. P., Killeen, J., and Rosenbluth, M. N.: 1963, Phys. Fluids 6, 459.

Gopasyak, S. I.: 1961, Soviet Astron. 5, 158.

Howard, R. and Severny, A. B.: 1963, Astrophys. J. 137, 1242.

Jaggi, R. K.: 1963, J. Geophys. Res. 68, 4429.

Leighton, R. B.: 1959, Astrophys. J. 130, 366.

Malville, J. M. and Tandberg-Hanssen, E.: 1969, Solar Phys. 6, 278.

Mayfield, E. B., Higman, J., and Samson, C.: 1970, Solar Phys. 13, 372.

Mogilevsky, E. I. and Shelting, B. D.: 1966, Atti del Convegno Sui Campi Magnetici Solari, (ed. by M. Cimino), G. Barbera, Firenze.

Petschek, H. E.: 1964, AAS/NASA Symposium on the Physics of Solar Flares, Washington, D.C.

Severny, A. B.: 1958, Soviet Astron. 2, 310.

Severny, A. B.: 1961, Soviet Astron. 5, 299. 
Severny, A. B.: 1963, Soviet Astron. 6, 747.

Severny, A. B.: 1969, in Annals of the IQSY, MIT Press, Cambridge, Mass., 3, 11.

Simon, G. W. and Leighton, R. B.: 1964, Astrophys. J. 140, 1120.

Sturrock, P. A.: 1968, in K. O. Kiepenheuer (ed.), 'Structure and Development of Solar Active Regions', IAU Symp. 35, 471.

Sturrock, P. A. and Coppi, B.: 1966, Astrophys. J. 143, 3.

Syrovat-skii, S. J.: 1966, Soviet Phys. JETP 23, 754.

\section{Discussion}

Deubner: How large was the area actually included in the analysis shown in the histograms?

Mayfield: The area analyzed was $190 \times 250$ arc s.

Wiehr: Your third and fourth observations were carried out far from the disk center, where the Leighton technique does not lead to the normal field component. A correction of this effect would require the knowledge of the transverse field component. If you did not consider this for your flux determinations I would suggest that the flux variations are not realistic.

Mayfield: Corrections for foreshortening were made. Changes in flux may be due to growth or decay of the magnetic region, changes between the longitudinal and transverse components and to loss to the flare. The observed change in flux is assumed to be associated with the flare but this cannot be proved.

Cowling: Decreases in magnetic flux should occur only through magnetic arches being drawn below the photosphere. Is there any sign of magnetic regions of opposite polarity flowing together?

Mayfield: Yes. We have observed such flows in time-lapse magnetograms where in complex fields small regions of opposite polarity merge. These will be shown by Vrabec later. 